\title{
Apfelallergie ist nicht gleich Apfelallergie
}

\author{
Eine Nahrungsmittelallergie entwickelt sich entweder aus \\ einer direkten Sensibilisierung oder sekundär als Kreuzallergie. \\ Ein schönes Beispiel für diese beiden Wege bieten aktuelle \\ Daten zum Sensibilisierungsweg bei Apfelallergie.
}

\begin{abstract}
$A^{n}$ $\mathrm{n}$ vier allergologischen Zentren in Europa wurden 389 Patienten mit einer Apfelallergie rekrutiert. Es erfolgten RASTs gegen das Birkenmajorallergen Bet $\mathrm{v} 1$ und die vier Apfelallergene Mal d 1, 2, 3 und 4. Mal d 1 ist immunologisch homolog $\mathrm{zu}$ Bet v 1, Mal d 2 gehört zur Pflanzenallergengruppe der Thaumatin-ähnlichen Proteine (TLP), Mal d 3 ist als nicht spezifisches Lipid-Transfer-Protein (nsLTP) Angehöriger einer Gruppe von Pflanzenproteinen/-allergenen mit hoher Stabilität im Gastrointestinaltrakt
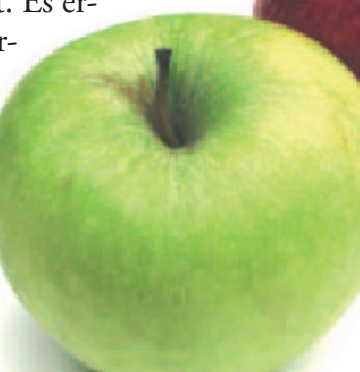

Patienten meist nur über eine milde Symptomatik, zu über 90\% treten lediglich isolierte lokale orale Reaktionen auf. Diese Allergie korreliert mit einer Birken-Pollinosis und einer Bet-v-1-Sensibilisierung sowie einer Sen-
\end{abstract}

sibilisierung gegen das Bet-v-1-Homolog Mal d 1. Bei diesen Patienten dürfte primär eine Pollenallergie vorgelegen haben, die sich zur Nahrungsmittelallergie weiterentwickelt hat.

In Spanien dagegen sind die Symptome der Apfelallergie meist schwer, bei 35\% der Patienten treten systemische Reaktionen auf. Diese Allergie korreliert mit einer Pfirsichallergie und einer Sensibilisierung gegen das nsLTP Mal d 3, das eine hohe Kreuzreaktivität zum Pfirsichhauptallergen Pru p 3 zeigt.

Fazit: Während bei einer Apfelallergie mit einer Sensibilisierung gegen Mal d 1 meist milde, primär orale Symptome dominieren, liegt in Spanien dagegen oft eine Sensibilisierung gegen Mal d 3 mit systemischen Reaktionen vor. $\quad b k$

Fernández-Rivas $\mathbf{M}$ et al. Apple allergy across Europe: How allergen sensitization profiles determine the clinical expression of allergies to plant foods. J Allergy Clin Immunol 2006; 118: 481-8

\section{Wieviel Erdnuss darf es höchstens sein?}

\section{Allergien gegen Erdnüsse sind für einen Großteil tödlich ver- laufender allergischer Reaktionen verantwortlich. In einer Studie sollten Schwellenwerte für eine unbedenkliche Erdnussexposition ermittelt werden.}

$\mathrm{N}$ iederländische Allergologen wollten die für Erdnussallergiker als unbedenklich geltende Erdnussdosis („no-observed-adverse-effect level“, NOAEL) bzw. die allergische Reaktionen hervorrufende Menge („eliciting dose“, ED) ermitteln. Dazu unterzogen sie 27 Erdnuss-sensibilisierte Kinder im Durchschnittsalter von 7,5 Jahren doppelblinden plazebokontrollierten $\mathrm{Nah}$ rungsmittelprovokationen (DBPCFC). Die Kinder erhielten neun Provokationsdosen zwischen $10 \mu \mathrm{g}$ und $3 \mathrm{~g}$ Erdnussmehl. Entsprechend den dabei beobachteten allergischen Reaktionen erhielten die Eltern anschließend Ernährungsratschläge und wurden gebeten, das Ernährungsverhalten und potenzielle aller- gische Reaktionen über zwölf Monate zu dokumentieren.

Bei 22 der Kinder (81\%) verlief der Provokationstest positiv. Der NOAEL betrug in dieser Kohorte $1 \mathrm{mg}$ Erdnussmehl, entsprechend $2 \mathrm{mg}$ ganze Erdnüsse. Die ED für das Auftreten von subjektiven Symptomen wie oralem Allergiesyndrom, Übelkeit und Leibschmerzen lag mit $10 \mathrm{mg}$ bis $3 \mathrm{~g}$ Erdnussmehl signifikant unter der für das Auftreten von objektiven Symptomen wie Urtikaria, Dyspnoe, Bronchokonstriktion oder Tachykardie (100 mg bis $3 \mathrm{~g}$; $\mathrm{p}=0,002$ ). Die ED korrelierte nicht mit den anamnestischen Angaben oder den Ergebnissen von Pricktest und Bestimmung des spezifischen $\operatorname{IgE}$.
Elf der Kinder mit einem positiven DBPCFC wurden ermahnt, auf eine strikt Erdnuss-freie Ernährung zu achten, bei elf weiteren Kindern waren die Einschränkungen nicht ganz so rigoros. Zehn Elternpaare dieser Kinder berichteten nach einem Jahr, dass ihre Sprösslinge auch Produkte zu sich nähmen, die möglicherweise Erdnussspuren enthielten. Drei der Kinder hatten nach dem Verzehr von Produkten, auf deren Verpackung auf einen möglichen Erdnussgehalt hingewiesen worden war, leichte allergische Symptome erlitten.

Fazit: Der in dieser Studie ermittelte Unbedenklichkeitsgrenzwert für die $\mathrm{Zu}$ fuhr von Erdnüssen betrug $2 \mathrm{mg}$. Die Compliance mit den Vorschriften zur Ernussvermeidung war bei der Hälfte der Kinder nicht vollständig.

Flinterman AE et al. Determination of noobserved-adverse-effect levels and eliciting doses in a representative group of peanut-sensitized children. J Allergy Clin Immunol 2006; 117: 448-54 